\title{
Genetic testing for hemochromatosis: Diagnostic or confirmatory test for iron overload?
}

\author{
Paul C Adams MD FRCPC
}

A fter the introduction of a simple genetic test for the C282Y mutation of the hemochromatosis gene (HFE) in 1997, many clinicians expected the diagnosis to become simpler and, perhaps, within the realm of family physicians. However, the widespread use of genetic testing for $\mathrm{C} 282 \mathrm{Y}$ and $\mathrm{H} 63 \mathrm{D}$ mutations has, in some ways, obscured the diagnosis of iron overload, and the interpretation of the genetic test may have led to more specialist consultation.

In the current issue of the Journal, Lanktree et al (1) (pages 41-45) reviewed the diagnostic yield of genetic testing for hemochromatosis at a McMaster (Hamilton, Ontario) medical centre from 664 patients submitted in 2012. Phenotypic information was available for only 160 patients, and the authors observed that only $18 \%$ of the referred patients were C282Y homozygotes or compound heterozygotes (C282Y/ H63D). The authors also advocate for a trial of phlebotomy in all patients with elevations in transferrin saturation (TS) and ferritin level. It appears from their data that many clinicians use the genetic test result to guide the need for phlebotomy.

In the early days of the genetic test, population screening strategies were developed, including the Hemochromatosis and Iron Overload Screening Study (HEIRS) (2,3). It had often been considered that a two-stage approach with a screening test (TS and or serum ferritin) followed by a confirmatory genetic test (C282Y homozygote) would be optimal. This would allow for non-HFE iron overload patients to be identified and treated, and would also minimize the potentially adverse effects of genetic testing in a healthy population. There were several unanticipated results from the HEIRS study, which screened 101,168 participants from a wide range of ethnic populations. There are a large number of patients without iron overload who have elevations in serum ferritin levels and TS. The ratio of elevated TS and ferritin in nonhomozygotes to homozygotes was 6.3. Non-HFE iron overload was almost nonexistent in this sample of $>100,000$ participants. There were no non-C282Y homozygotes that had $>4 \mathrm{~g}$ of body iron stores removed (4). Elevations in serum ferritin levels were more common in Asians than Caucasians and, yet, there were no documented cases of iron overload in Asians (5). This background noise of high iron tests without iron overload led to a diagnostic phone app and website based on regression analysis in 44,809 Caucasians from the HEIRS study. For example, using this calculator, a Caucasian man with a ferritin level of $500 \mu \mathrm{g} / \mathrm{L}$ and a TS of $50 \%$ has only a $1.3 \%$ probability of being a C282Y homozygote (6).

The authors imply that more diligence about restricting genetic testing to patients with elevations in TS and serum ferritin levels would increase the number of participants with a positive genetic test. This is a correct prediction; however, the percentage of patients who are C282Y homozygotes, even with an elevated TS and ferritin levels in Caucasians, is often $<50 \%$ (Figure 1). This is a yield similar to other confirmatory tests in medicine such as an anti-DNA test for systemic lupus erythematosus in a patient with a positive antinuclear antigen. Minimizing the genetic test to reduce costs is an issue of cost versus charges. The cost for the reagents for a single-mutation gene test are lower than the costs for a serum transferrin plus serum ferritin study.

The final issue is whether clinicians should use the genetic test information to decide on the need for phlebotomy. This was apparent in the McMaster study and in the HEIRS study because $70 \%$ of C282Y homozygotes were treated compared with only $11 \%$ of nonhomozygotes (4). In my own clinical practice, I use the genetic test result to guide the need for phlebotomy because I do not believe that most of the other cases have iron overload. To determine whether they have iron overload can be invasive (liver biopsy), insensitive (magnetic resonance imaging) or after the fact (trial of phlebotomy). It has been noted that if a patient is referred to a hematology clinic, they may be told they have iron overload and undergo a trial of phlebotomy. If the patient is referred to a liver specialist, they may undergo further testing such as a liver biopsy, and are told that they do not have iron overload and do not need phlebotomy. Another approach is to suggest for a patient with mild abnormalities in serum ferritin and TS to become a voluntary blood donor every two months. This approach introduces the paradox that, if you believe the ferritin level is elevated because of inflammation, why would you recommend blood donation, and why would the ferritin level decline after blood donation? The answer was demonstrated recently in a study with serial liver biopsies after phlebotomy for fatty liver, in which the liver iron concentration decreased from the middle normal range to low normal range during the phlebotomy program (7). There are different approaches and solutions to these common clinical problems. The future may include multigene genetic panels; however, the interpretation of these results will require tertiary care consultation and, in some cases, ambiguous genetic test results increases anxiety and health care costs (8).

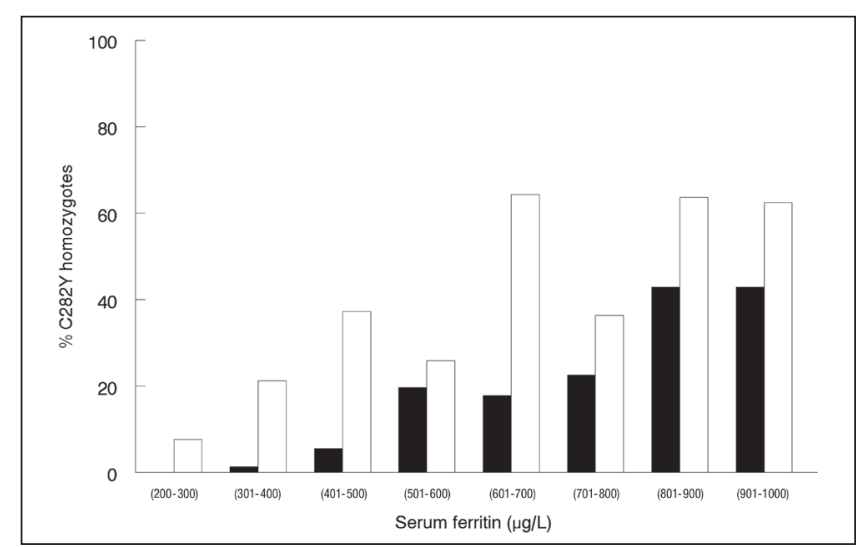

Figure 1) The proportion of male (black bars [n=378]) and female (open bars $[n=272])$ participants with an elevated ferritin level and transferrin saturation who were C282Y homozygotes. Reproduced with permission from reference 9 


\section{REFERENCES}

1. Lanktree M, Lanktree B, Pare G, et al. Examining the clinical use of hemochromatosis genetic testing. Can J Gastroenterol Hepatol 2015;29:41-5.

2. Adams PC. Population screening for haemochromatosis. Gut 2000;1;46:301-3.

3. Adams PC, Reboussin DM, Barton JC, et al. Hemochromatosis and iron-overload screening in a racially diverse population.

N Engl J Med 2005;352:1769-78.

4. Gordeuk VR, Reboussin DM, McLaren CE, et al. Serum ferritin concentrations and body iron stores in a multicenter, multiethnic primary-care population. Am J Hematol 2008;83:618-26.

5. Harris EL, McLaren CE, Reboussin DM, et al. Serum ferritin and transferrin saturation in Asians and Pacific Islanders. Arch Intern Med 2007;167:722-6.
6. Lim A, Speechley M, Adams PC. Predicting C282Y homozygote genotype for hemochromatosis using serum ferritin and transferrin saturation values from 44,809 participants of the HEIRS study. Can J Gastroenterol Hepatol 2014;28:502-4.

7. Beaton MD, Chakrabarti S, Adams PC. Inflammation is not the cause of an elevated serum ferritin in non-alcoholic fatty liver disease. Ann Hepatol 2014;13:353-6.

8. Speechley M, Alter D, Guo H, et al. Effect of ambiguous hemochromatosis gene test results on physician utilization. Med Care 2012;50:394-8.

9. Adams PC, McLaren CE, Speechley M, et al. HFE mutations in Caucasian participants of the Hemochromatosis and Iron Overload Screening study with serum ferritin level $<1000 \mu \mathrm{g} / \mathrm{L}$. Can J Gastroenterol 2013;27:390-2. 


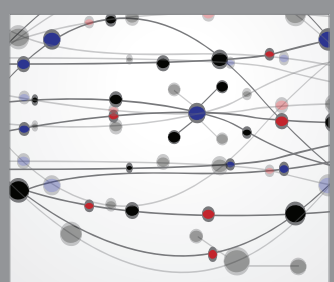

The Scientific World Journal
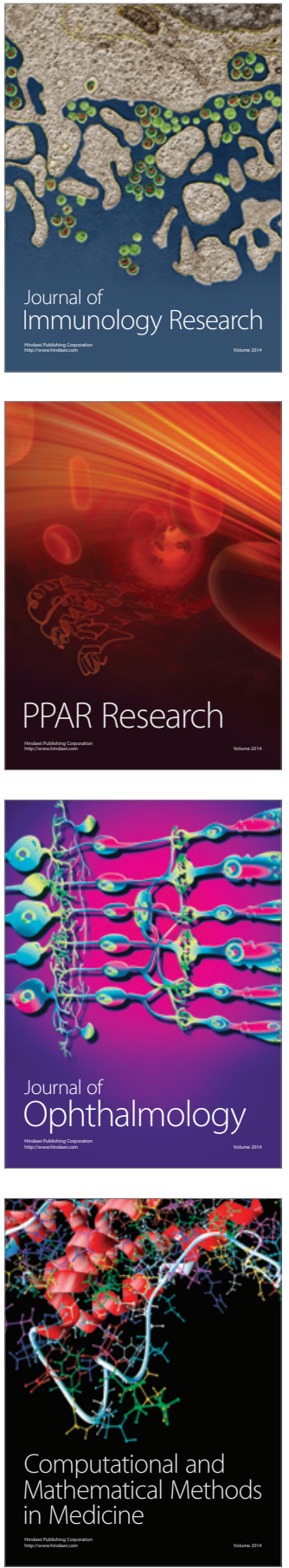

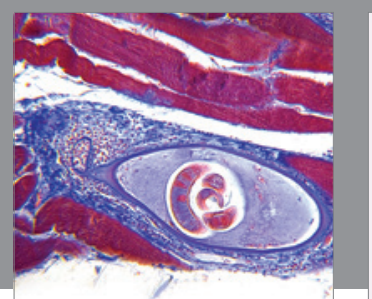

Gastroenterology Research and Practice

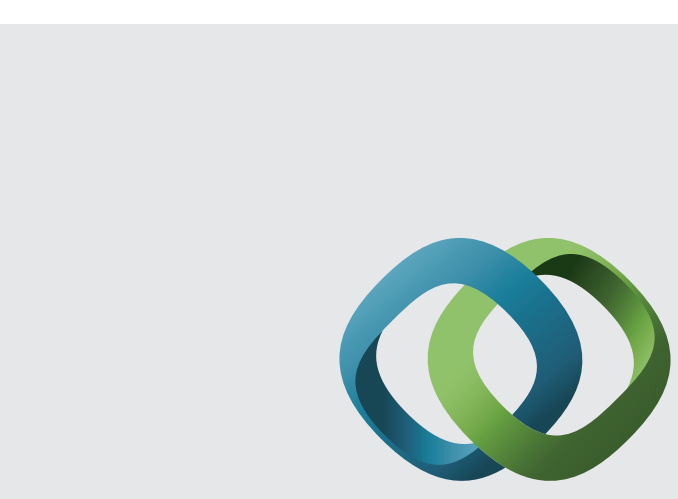

\section{Hindawi}

Submit your manuscripts at

http://www.hindawi.com
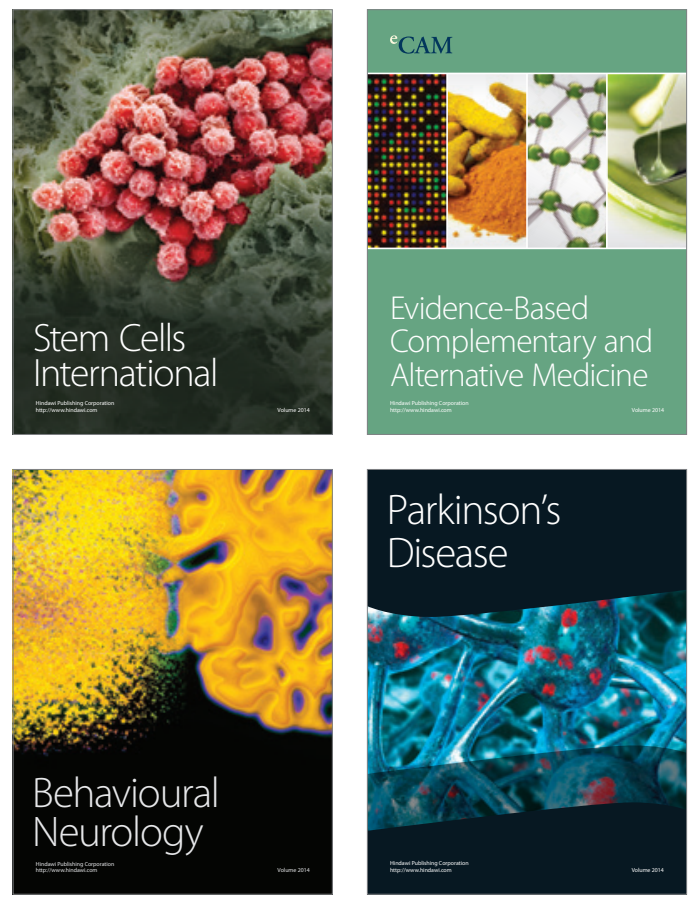
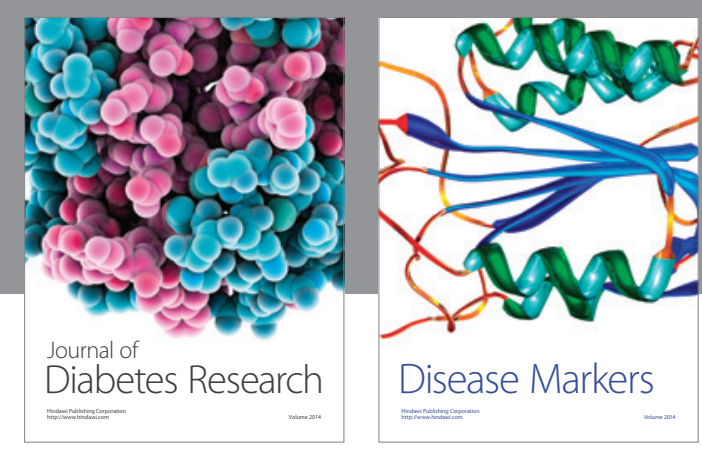

Disease Markers
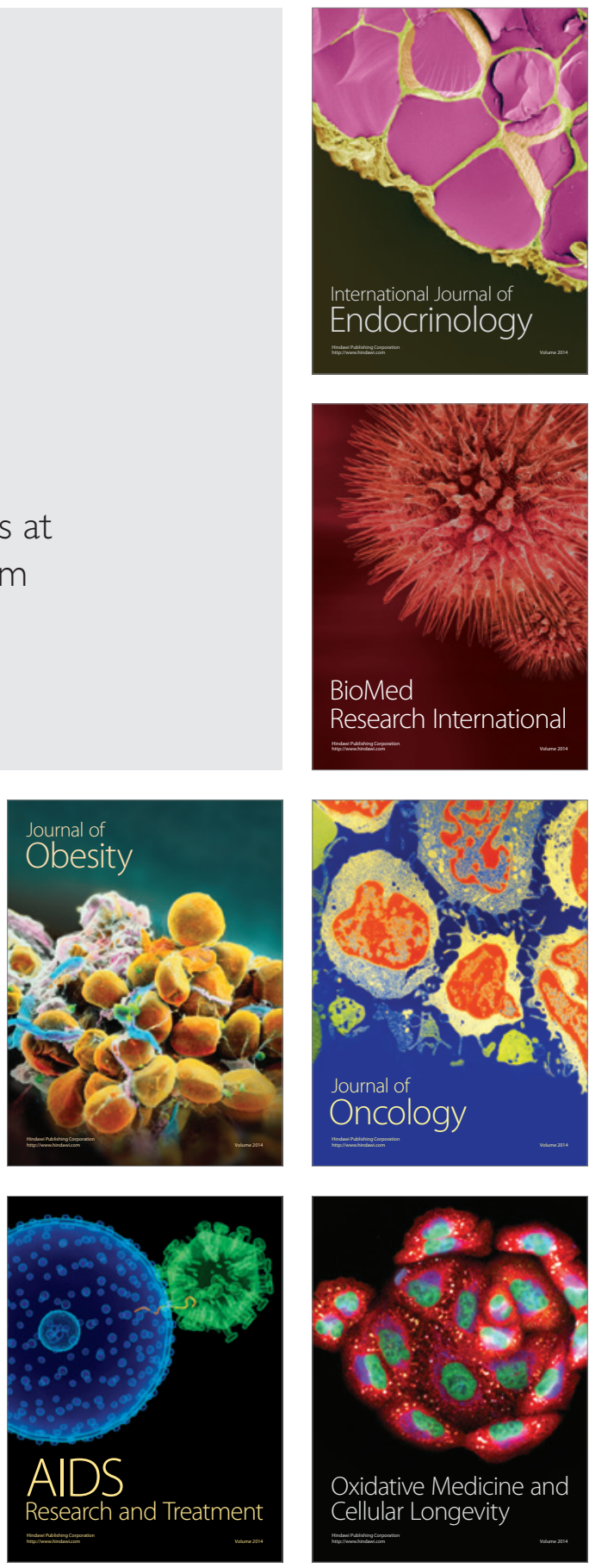